\title{
Prebiotics and probiotics in atopic dermatitis (Review)
}

\author{
EMILIA RUSU ${ }^{1}$, GEORGIANA ENACHE $^{1,2}$, RALUCA CURSARU $^{1}$, ALEXANDRA ALEXESCU $^{1}$, \\ RALUCA RADU $^{1}$, OANA ONILA ${ }^{1}$, TEODORA CAVALLIOTI ${ }^{1}$, FLORIN RUSU ${ }^{3}$, \\ MIHAELA POSEA $^{4}$, MARIANA JINGA ${ }^{5}$ and GABRIELA RADULIAN ${ }^{1}$ \\ ${ }^{1}$ Department 2 Infectious Diseases, Epidemiology, Microbiology, Parasitology, Virology, Diabetes, Endocrinology, \\ 'Carol Davila' University of Medicine and Farmacy, 010458 Bucharest; ${ }^{2}$ Department of Diabetes, Nutrition and \\ Metabolic Diseases, 'Dr. Pompei Samarian' County Emergency Hospital, 910071 Calarasi; ${ }^{3}$ Department of Urology, \\ 'Dr. Carol Davila' Central Military Emergency University Hospital, 010825 Bucharest; ${ }^{4}$ Department of \\ Diabetes, Nutrition and Metabolic Diseases, Smart Nutrition Clinic, 051075 Bucharest; ${ }^{5}$ Department of \\ Gastroenterology, 'Dr. Carol Davila' Central Military Emergency University Hospital, 051075 Bucharest, Romania
}

Received September 21, 2018; Accepted March 1, 2019

DOI: $10.3892 / \mathrm{etm} .2019 .7678$

\begin{abstract}
Recent years have brought additional data on the benefits of prebiotics and probiotics treatment in patients with atopic dermatitis. This review includes all the articles published in PubMed, Scopus, Embase and Cochrane databases until 05.06.2018. The terms used for the search were 'prebiotic', 'probiotic', 'atopic dermatitis', 'Severity Scoring of Atopic Dermatitis', and 'SCORAD'. There was an increase of the intestinal permeability reported in patients with atopic dermatitis and a reduction of the cutaneous microbiome diversity. Probiotics modulate the general microbiome and immune status by improving the intestinal barrier; these effects can be responsible for reducing allergic phenomenon and atopic dermatitis severity. We have structured the results by age groups as infants, 1-18 years, adults, and also pregnancy and lactation. Literature does not offer yet answers on the issues such as the optimal dosing, duration needed to see beneficial effects, the optimal time to start the treatment; the personalized use of probiotics according to colonic
\end{abstract}

Correspondence to: Dr Georgiana Enache, Department of Diabetes, Nutrition and Metabolic Diseases, 'Dr. Pompei Samarian' County Emergency Hospital, 1-3 Eroilor Street, 910071 Calarasi, Romania

E-mail: georgianamd@gmail.com

Abbreviations: AD, atopic dermatitis; CD14, cluster of differentiation 14; GM, gut microbiome; HDL, high-density lipoprotein; hs-CRP, high-sensitivity $\mathrm{C}$ reactive protein; IgA, immunoglobulin A; IL, interleukin; INF, interferon; IR, insulin resistance; LPS, lipopolysaccharide; SCFAs, short-chain fatty acids; SCORAD, severity scoring of atopic dermatitis; Th1, T-helper cell type-1; Th2, T-helper cell type-2; Treg, regulatory T cell; TLR4, Toll-like receptor 4 ; TGF- $\beta$, transforming growth factor- $\beta$; TNF- $\alpha$, tumor necrosis factor- $\alpha$; WAO, World Allergy Organisation

Key words: prebiotic, probiotic, atopic dermatitis, SCORAD dysbiosis may be associated with better results. However, most randomized controlled trials and meta-analyzes support the administration of probiotics for at least 8 weeks in order to obtain beneficial effects in improving severity scoring of atopic dermatitis.

\section{Contents}

1. Introduction

2. Definition of terms

3. Modulating the human microbiome by pre- and probiotics

4. Prebiotic and probiotic in atopic dermatitis

5. The type of probiotics used in clinical trials

6. Prebiotics - mechanism of action

7. Probiotics - mechanism of action

8. Conclusion

\section{Introduction}

Food can have therapeutic benefits; as early as 2500 years ago the parent of medicine, Hippocrates said: 'Let food be thy medicine and medicine be thy food'.

Atopic dermatitis (AD) is a chronic, inflammatory skin disease, characterized by dry skin, intense pruritus, eczematous cutaneous lesions that occurs in people with atopic terrain. It is manifested by intense pruritic papules with evolution towards lichenification. Patients with AD may associate other atopy (allergic rhinitis, bronchial asthma). AD prevalence is increasing, reaching $10-20 \%$ in the pediatric population and up to $3 \%$ of adults, commonly the onset occurs during the first year of life (1).

Etiopathogeny of AD is complex and multifactorial involving genetic factors, environmental factors and immunological factors. The composition of the intestinal and cutaneous microbiome, the maternal diet during pregnancy, the mode of delivery, antibiotic treatment during pregnancy and early infancy, westernized lifestyle with chronic exposure to allergens increase the risk of allergic diseases and AD. 
The severity of AD can be assessed using the SCORAD severity score (Severity Scoring of Atopic Dermatitis) (2).

\section{Definition of terms}

Prebiotics are non-digestible ingredients that beneficially affect the host by selectively stimulating growth or limiting some species of intestinal bacteria, such as Bifidobacterium and Lactobacilli that have the potential to improve the health of the host (3).

Probiotics are living microorganisms that confer a health benefit on the host when there are administered in adequate amounts (4).

The most known microorganism used as probiotics are the bacteria from the Lactobacillus family (acidophilus, sporogenes, lactis, reuteri RC-14, GG, L. plantarum 299v), Bifidobacterium (bifidum, longum, infantis), Streptococcus group (thermophillus, lactis, fecalis), but there are also nonbacterial organisms (non-pathogenic yeast Saccharomyces boulardii). Lactobacillus and Bifidobacteria belong to normal microbial flora; they are Gram-positive, anaerobic bacteria, and several types produce not only lactic acid but also other antimicrobial substances such as hydrogen peroxide and bacteriocins (small proteins that may have a bactericidal effect) (5).

\section{Modulating the human microbiome by pre- and probiotics}

Gut microbiome (GM) is the genetic material of all microbes, for example bacteria, fungi, protozoa and viruses living on and inside the digestive tract of humans and other animals, including insects. The composition of human microbiome varies depending on age, sex, and exposure to antibiotics.

Bacteria are the dominant microorganisms in the colon (over 1,014 belonging to $\sim 500-1,000$ distinct species). The predominant bacteria in the gut microbiome in adult population in Europe are Bacteroidetes, Firmicutes, Actinobacteria, Proteobacteria and Verrucomicrobia. Human intestinal microbiome is closely related to the host and there are no two human beings with identical intestinal microbiome.

The functions of the intestinal microbiome. Gut microbiome provides numerous biological and metabolic functions; the alteration of the intestinal bacteria balance may initiate inflammation through the activity of lipopolysaccharide (LPS), a component of the cell wall of gram-negative bacteria that triggers the inflammatory process by binding to the CD14/ Toll-like receptor 4 (TLR-4) complex. GM may influence the metabolism of the host by modulating the tissue composition of fatty acids; the species of Lactobacilli and Bifidobacteria produce bioactive isomers of conjugated linoleic acid with immunomodulating properties, reducing the proinflammatory cytokines. Additionally, intestinal microbiome synthesizes a significant amount of glycosidic hydrolases that decompose plant complexes into monosaccharides and short chain fatty acids (SCFAs) (acetate, propionate, butyrate). SCFAs play an important role in energy metabolism; butyrate provides energy for colonic epithelial cells; propionate affects lipogenesis and hepatic gluconeogenesis, while acetate, at the peripheral level, functions as a substrate for cholesterol synthesis. The mucosal colon depends on the presence of butyrate as a source of energy on the lumen level, and the lack of these SCFAs is considered to play an important role in the pathogenesis of intestinal disease and inflammatory bowel disease.

Probiotics modulate the intestinal microbiome and immune status by improving the intestinal barrier; these effects are responsible for reducing allergic phenomenon and AD severity (6).

Intestinal permeability is increased in AD patients (7). In addition, babies born by caesarean section have a lower colonization with Bacteroides and higher with Clostridium (8). Also, early colonization with Escherichia coli has a protective role for $\mathrm{AD}(9)$.

The skin microbiome and atopic dermatitis. There are 4 strains prevailing on the skin surface: Firmicutes (Staphylococcus, Streptococcus, Anaerococcus, Finegoldia, Veillonella, Lactobacillus, Peptoniphilus), Actinobacteria (Propionibacterium, Corynebacterium, Micrococcus, Kocuria, Actinomyces, Rothia), Proteobacteria (Acinetobacter, Haemophilus, Enhydrobacter, Neisseria, Microvirgula), and Bacteriodetes (Prevotella, Chryseobacterium, Fusobacteria, Leptotrichia). The most common genus is Staphylococcus; within the Staphylococcus genus, the most common species in healthy skin is Staphylococcus epidermidis (10-12).

A reduction of the cutaneous microbiome diversity was reported in AD patients (13), AD being associated with early colonization with Staphylococcus aureus. The cutaneous presence of Staphylococcus epidermidis and Staphilococcus cohnii (11) has proven a protective effect against AD in children. Increased colonies of S. aureus, S. epidermidis, Propionibacteria, Corynebacteria and Streptococcus (11) were isolated in the atopic dermatitis lesions. Another study has found an association between AD severity and the abundance of the genus Corynebacterium and the phylum Proteobacteria. The presence and chronicity of eczema appear to be more important determinants of skin microbiome configuration $(14,15)$.

Other studies have shown that there is also an increase in fungal diversity, as Malassezia restricta, Malassezia globosa and Malassezia dermatis have been isolated in $~ 90 \%$ of patients with atopic dermatitis (16). Several studies have described M. sympodialis as the most abundant in patients with atopic dermatitis and the possibility of its coexistence with a non-Malassezia species called Cryptococcus diffluens (17). In addition, many patients with AD have associated IgE mediated sensitization to Malassezia species, with positive skin prick tests $(18,19)$. The rate of IgE mediated Malassezia sensitization correlates with disease severity $(18,20)$.

The presence of Demodex mites (Demodex folliculorum and Demodex brevis) was not associated with an increased prevalence of $\mathrm{AD}(21)$.

Changes in skin microbiome composition were also observed in other dermatological conditions such as rosacea, acne, psoriasis, or seborrheic dermatitis; Demodex folliculorum, Bacillus oleronius, Helicobacter pylori, Staphylococcus epidermidis and Chlamydophila pneumonia were associated with rosacea $(14,22-24)$. In adolescence and adults with acne in the affected areas there were mostly lipophilic Propionibacterium (15). 


\section{Prebiotic and probiotic in atopic dermatitis}

In the past few years, more ongoing studies evaluated the administration of pre- and probiotics in patients with atopic dermatitis.

Prebiotics and probiotics in pregnancy and lactation. Preventive/prophylactic avoidance of major allergens during pregnancy and lactation does not protect against AD development during the first 18 months of life (results from a systematic review that included 5 clinical trials, 952 participants) (25).

World Allergy Organisation (WAO) does not recommend the use of prebiotics neither during pregnancy nor during lactation as a preventive measure against atopic dermatitis for lack of solid scientific evidence (26).

In a meta-analysis led by Pelucchi et al (1,619 patients treated/1,475 placebo), there was a modest role of diet supplementation with probiotics in pregnancy to prevent $\mathrm{AD}$ in infants (27).

In another meta-analysis published in 2012, conducted by Doege et al (28), that included seven randomised, double-blind, placebo-controlled trials, only the supplementing with Lactobacilli during pregnancy prevented AD in children aged 2-7 years; other probiotic supplements, with or without Lactobacilli, have proven no favorable results (28).

The beneficial effects of the administration of probiotics during pregnancy have been reconfirmed by a recent meta-analysis; this meta-analysis that included over 19 trials (4,076 persons exposed to probiotics/3,700 control group) reported the effect of probiotic consumption during the last part of pregnancy and during the lactation period to decrease AD incidence in children under five years (29).

In some studies, the administration of probiotics started during the first trimester, or after the 36th week of gestation, and continued during breastfeeding (27-29).

Prebiotics and probiotics in infants. Prebiotics administered in the first year of life reduce the risk of asthma or food allergy but the results on atopic dermatitis were inconclusive $(26,30)$.

A systematic review of a meta-analysis that included 8 clinical trials totaling 741 infants, demonstrated the beneficial effect of Lactobacillus-containing probiotics on AD severity reduction (31). Probiotics with Bifidobacterium (3 studies, 73 infants) did not prove beneficial effects; this result should be interpreted with caution due to the small number of subjects included and the heterogenity of the studies. Most studies included in meta-analysis followed patients for a limited time (under 8 weeks). Data previously published revealed a smaller number of Bifidobacteria strains in the children's feces with atopic dermatitis $(32,33)$. However, the conclusion of this article was that infants with moderate and severe AD presented a protective effect of probiotics (28).

Prebiotics and probiotics in children (1-18 years). In a recent meta-analysis (568 children, 1-18 years; intervention group, 296; control group, 272), there was an improvement in SCORAD reported in children (1-18 years) with $\mathrm{AD}$ given probiotics (34). Lactobacillus fermentum, Lactobacillus and a mixture of different strains (Bifidobacterium bifidum, Lactobacillus acidophilus, Lactobacillus casei and Lactobacillus salivarius) improved significantly the SCORAD values in children (34). These data reconfirm the results of the previously published studies (35).

A recent randomized, double-blind, placebo-controlled intervention trial, that included 50 children aged between 4-17 years, reported that the mixture of probiotics (Bifidobacterium lactis CECT 8145, Bifidobacterium longum CECT 7347, and Lactobacillus casei CECT 9104) was effective in reducing SCORAD index (36). The probiotic was administered between 4-12 weeks.

Prebiotics and probiotics in adult. We found several randomized clinical trials and one meta-analysis that have reported beneficial effects of probiotics in AD adult patients (35).

Roessler et al (37) in a double-blind, placebo-controlled, randomized cross-over study, using a combination of the probiotics (Lactobacillus paracasei Lpc-37, Lactobacillus acidophilus 74-2, and Bifidobacterium animalis subsp. lactis DGCC 420) for 8 weeks, observed that the SCORAD tended to decrease. There was an increased number of $L$. paracase $i$ and B. lactis isolated in $\mathrm{AD}$ patient's faeces after supplementation.

Another study conducted by Yoshida et al that used Bifidobactrium breve for 8 weeks in AD patients reported that the severity scoring for atopic dermatitis significantly improved and the proportion of $B$. breve in intestinal microflora was increased in the probiotic group (38).

Drago et al using L. salivarius for 16 weeks in 38 patients with moderate to severe AD reported an improvement in SCORAD and a significant decrease of T-helper cell type-1 (Th1) cytokines [interleukin-12 (IL-12), interferon (INF)- $\gamma]$ and Th1/Th2 (IL-12, IFN- $\gamma /$ IL-4, IL-5) ratio (39); they also reported a significant reduction of staphylococci in faeces in the probiotic treated group.

Iemoli et al (40) using a specific mixture of probiotics (L. salivarius LS01/B. breve BR03) for 12 weeks in 48 adults suffering from moderate to severe AD reported an improvement in SCORAD and of the immunological profile [Th1/ Th2 ratio, T-helper cell 17/regulatory $\mathrm{T}$ cell (Treg) ratio] and a reduced microbial translocation.

Matsumoto et al (41) reported that oral intake of Bifidobacterium animalis subsp. lactis LKM512 may exert antipruritic effects in adults with atopic dermatitis.

Although these studies are promising, the small number of subjects is limiting the generalization of the results.

\section{The type of probiotics used in clinical trials}

Probiotic micro-organisms must be appropriate (non-pathogenic and non-toxic) for human consumption. Toxicity tests are conducted as for pharmaceuticals. They must not affect taste, texture, and they must survive in the probiotic food in a sufficiently large concentration until they are consumed.

Various studies conducted in patients with atopic dermatitis evaluated the probiotics such as lactobacilli, bifidobacteria or combinations (Table I).

\section{Prebiotics - mechanism of action}

Prebiotics enhance the production of SCFAs (acetate, propionate, and butyrate) which have anti-inflammatory effects (42), 
Table I. Species of probiotic bacteria used in atopic dermatitis (adapted after refs. 36,37,49).

\begin{tabular}{lll}
\hline Lactobacillus & \multicolumn{1}{c}{ Bifidobacterium } & Saccharomyces \\
\hline L. rhamnosus GG & B. longum reuter & Saccharomyces boulardii \\
L. rhamnosus LC705 & B. longum infantis & \\
L. fermentum VRI-033 & B. breve & B. lactis UABLA-12 \\
L. paracasei & & \\
L. plantarum & & \\
L. salivarius & & \\
L. acidophilus &
\end{tabular}

reduce the generation of toxic fermentation products (43) and improve the Th1/Th2 ratio $(44,45)$, increased lymphocyte and/or leucocyte numbers in gut-associated lymphoid tissues (44), increased intestinal IgA secretion (44).

\section{Probiotics - mechanism of action}

Immunomodulatory effect. Probiotics can reduce the severity of AD by inhibiting the T-helper cell type-2 (Th2) mediated response and improving the Th1/Th2 ratio (46); inhibiting Th2 cell response, cytokines such as IL-4, IL-5, IL-6 and IL-13 are no longer released (47-49), INF- $\gamma$ decrease (cytokine released by Th1 cells), phagocytosis is stimulated, serum IgA is increased (50). Probiotics also stimulate the secretion of IL-10 and transforming growth factor- $\beta$ (TGF- $\beta$ ) (22). Probiotics can reduce inflammation by reducing proinflammatory cytokines, IL-4, IL-6, tumor necrosis factor- $\alpha$ (TNF- $\alpha$ ), INF- $\gamma$ and high sensitivity $\mathrm{C}$ reactive protein (hsCRP) (51) and by increasing expression of IL-10 and Treg-related cytokines at mesenteric lymph nodes. A new mechanism proposed to demonstrate the effectiveness of probiotics is the inhibition of the mature dendritic cell differentiation and transformation of naive $\mathrm{T}$ cells into Th2 (52). Immunomodulation decreases the susceptibility to inflammatory and allergic factors modulating the intestineskin axis. Probiotics also modulate brain function including stress response on the intestine-brain axis (53).

Normalization of microbial composition provides protection against pathogens at the mucosal surface. In newborns, the distribution of different Bifidobacterium species in the faeces influences the prevalence of allergic diseases. In a study that proposed to detect differences in levels of different Bifidobacterium species in faeces of children with allergies compared to healthy ones, significantly higher levels of Bifidobacterium longum were isolated in healthy children, suggesting the role of this strain in preventing the occurrence of bronchial asthma and allergic dermatitis (54).

Metabolic effects. Medical nutrition therapy plays an important role in modulation of the intestinal microflora. The use of 'functional food' as prebiotics, probiotics, natural antioxidant $(55,56)$ was associated with good metabolic effects such as improving digestion and absorbtion of food ingredients and minerals, vitamin synthesis, and thereby improving overall nutritional status and health.
Probiotic consumption can be associated with reduction of blood glucose, insulinemia and insulin resistance (IR) (57). Some probiotic strains have antioxidant effects (58). A recent meta-analysis has shown favorable effects of alanine aminotransferase decrease and IR reduction in patients with non-alcoholic fatty liver disease (59).

In another meta-analysis, the administration of probiotics was associated with a decrease in total cholesterol, triglycerides and low-density lipoprotein (LDL)-cholesterol and increase in high-density lipoprotein (HDL)-cholesterol (60); beneficial effects on the lipid profile are secondary to reducing intestinal absorption of dietary cholesterol and suppressing bile acid reabsorption.

In a systematic review and meta-analysis, Zhang and Silverberg concluded that patients with overweight and obesity had a higher risk of AD; the association was significant in North America and Asia but not Europe (61). The major limitations of this meta-analysis are related to the cross-sectional design of the included studies. A study published in Germany, did not observe an increase in prevalence of $\mathrm{AD}$ in patients with metabolic syndrome (obesity, diabetes, hypertension and hyperlipidemia) (62).

The relationship between metabolic disorders (obesity, dyslipidemia) and atopic dermatitis can be mediated by chronic systemic inflammation, proinflammatory cytokines (IL-6, TNF- $\alpha$ and CRP), increased oxidative stress and consequent change in expression of inflammatory genes.

\section{Conclusion}

Improving nutritional status, nutrient digestion, specific and non-specific immune response, beneficial effects on the gastrointestinal tract and skin, are arguments for supporting the use of pre- and probiotics in patients with AD. Still, there is not enough data in the literature to respond to questions regarding optimal dosing, optimal time to start treatment and duration necessary to show beneficial effects; the personalized use of probiotics according to colonic dysbiosis can be associated with best results. However, most randomized controlled trials and meta-analyzes, support the administration of probiotics for at least 8 weeks with beneficial effects in improving severity scoring of atopic dermatitis.

\section{Acknowledgements}

Not applicable. 


\section{Funding}

No funding received.

\section{Availability of data and materials}

Not applicable.

\section{Authors' contributions}

ER and GR designed the study; ER, GE, RC, AA, RR, OO, TC, FR, MP, MJ and GR performed the literature search and article selection, and drafted the manuscript. The final version of the manuscript read and approved by all authors.

\section{Ethics approval and consent to participate}

Not applicable.

\section{Patient consent for publication}

Not applicable.

\section{Competing interests}

The authors declare that they have no competing interests.

\section{References}

1. Nutten S: Atopic dermatitis: Global epidemiology and risk factors. Ann Nutr Metab 66 (Suppl 1): 8-16, 2015.

2. Kunz B, Oranje AP, Labrèze L, Stalder JF, Ring J and Taïeb A: Clinical validation and guidelines for the SCORAD index: Consensus report of the European Task Force on Atopic Dermatitis. Dermatology 195: 10-19, 1997.

3. Gibson GR and Roberfroid MB: Dietary modulation of the human colonic microbiota: Introducing the concept of prebiotics. J Nutr 125: 1401-1412, 1995.

4. Hill C, Guarner F, Reid G, Gibson GR, Merenstein DJ, Pot B, Morelli L, Canani RB, Flint HJ, Salminen S, et al: The International Scientific Association for Probiotics and Prebiotics statement on the scope and appropriate use of the term probiotic. Nat Rev Gastroenterol Hepatol 11: 506-514, 2014.

5. Alvarez-Olmos MI and Oberhelman RA: Probiotic agents and infectious diseases: A modern perspective on a traditional therapy. Clin Infect Dis 32: 1567-1576, 2001.

6. Lin RJ, Qiu LH, Guan RZ, Hu SJ, Liu YY and Wang GJ: Protective effect of probiotics in the treatment of infantile eczema. Exp Ther Med 9: 1593-1596, 2015.

7. Isolauri E: Intestinal involvement in atopic disease. J R Soc Med 90 (Suppl 30): 15-20, 1997.

8. Penders J, Gerhold K, Thijs C, Zimmermann K, Wahn U, Lau S and Hamelmann E: New insights into the hygiene hypothesis in allergic diseases: Mediation of sibling and birth mode effects by the gut microbiota. Gut Microbes 5: 239-244, 2014.

9. Orivuori L, Mustonen K, de Goffau MC, Hakala S, Paasela M, Roduit C, Dalphin JC, Genuneit J, Lauener R, Riedler J, et al; PASTURE Study Group: High level of fecal calprotectin at age 2 months as a marker of intestinal inflammation predicts atopic dermatitis and asthma by age 6. Clin Exp Allergy 45: 928-939, 2015.

10. Iwase T, Uehara Y, Shinji H, Tajima A, Seo H, Takada K, Agata T and Mizunoe Y: Staphylococcus epidermidis Esp inhibits Staphylococcus aureus biofilm formation and nasal colonization. Nature 465: 346-349, 2010

11. Cogen AL, Yamasaki K, Muto J,SanchezKM, Crotty Alexander L, Tanios J, Lai Y, Kim JE, Nizet V and Gallo RL: Staphylococcus epidermidis antimicrobial delta-toxin (phenol-soluble modulin-gamma) cooperates with host antimicrobial peptides to kill group A Streptococcus. PLoS One 5: e8557, 2010.
12. Baurecht H, Rühlemann MC, Rodríguez E, Thielking F, Harder I, Erkens AS, Stölzl D, Ellinghaus E, Hotze M, Lieb W, et al: Epidermal lipid composition, barrier integrity, and eczematous inflammation are associated with skin microbiome configuration. J Allergy Clin Immunol 141: 1668-1676.e16, 2018.

13. Wollina U: Microbiome in atopic dermatitis. Clin Cosmet Investig Dermatol 10: 51-56, 2017.

14. Musthaq S, Mazuy A and Jakus J: The microbiome in dermatology. Clin Dermatol 36: 390-398, 2018.

15. Grice EA: The skin microbiome: Potential for novel diagnostic and therapeutic approaches to cutaneous disease. Semin Cutan Med Surg 33: 98-103, 2014.

16. Dima MB, Orzan OA, Popa LG, Dima B and Mihai MM: The altered skin microbiota - key role in atopic dermatitis? Infectio 53: $12-15,2018$

17. Jagielski T, Rup E, Ziółkowska A, Roeske K, Macura AB and Bielecki J: Distribution of Malassezia species on the skin of patients with atopic dermatitis, psoriasis, and healthy volunteers assessed by conventional and molecular identification methods. BMC Dermatol 14: 3, 2014.

18. Fenner J and Silverberg NB: Skin diseases associated with atopic dermatitis. Clin Dermatol 36: 631-640, 2018.

19. Johansson C, Eshaghi H, Linder MT, Jakobson E and Scheynius A: Positive atopy patch test reaction to Malassezia furfur in atopic dermatitis correlates with a T helper 2-like peripheral blood mononuclear cells response. J Invest Dermatol 118: 1044-1051, 2002.

20. Glatz M, Buchner M, von Bartenwerffer W, SchmidGrendelmeier P, Worm M, Hedderich J and Fölster-Holst R: Malassezia spp.-specific immunoglobulin E level is a marker for severity of atopic dermatitis in adults. Acta Derm Venereol 95: 191-196, 2015.

21. Tehrani S, Tizmaghz A and Shabestanipour G: The Demodex mites and their relation with seborrheic and atopic dermatitis. Asian Pac J Trop Med 7S1: S82-S84, 2014.

22. Tatu AL, Ionescu MA, Clatici VG and Cristea VC: Bacillus cereus strain isolated from Demodex folliculorum in patients with topical steroid-induced rosaceiform facial dermatitis. An Bras Dermatol 91: 676-678, 2016.

23. Tatu AL, Clatici VG and Nwabudike LC: Rosacea-like demodicosis (but not primary demodicosis) and papulopustular rosacea may be two phenotypes of the same disease - a microbioma, therapeutic and diagnostic tools perspective. J Eur Acad Dermatol Venereol: Jun 29, 2018 (Epub ahead of print). doi: 10.1111/ jdv.15166.

24. Tatu AL, Clatici V and Cristea V: Isolation of Bacillus simplex strain from Demodex folliculorum and observations about Demodicosis spinulosa. Clin Exp Dermatol 41: 818-820, 2016.

25. Kramer MS and Kakuma R: Maternal dietary antigen avoidance during pregnancy or lactation, or both, for preventing or treating atopic disease in the child. Cochrane Database Syst Rev 9: CD000133, 2012

26. Cuello-Garcia CA, Fiocchi A, Pawankar R, Yepes-Nuñez JJ, Morgano GP, Zhang Y, Ahn K, Al-Hammadi S, Agarwal A, Gandhi S, et al: World Allergy Organization-McMaster University Guidelines for Allergic Disease Prevention (GLAD-P): Prebiotics. World Allergy Organ J 9: 10, 2016.

27. Pelucchi C, Chatenoud L, Turati F, Galeone C, Moja L, Bach JF and $\mathrm{La}$ Vecchia C: Probiotics supplementation during pregnancy or infancy for the prevention of atopic dermatitis: A meta-analysis. Epidemiology 23: 402-414, 2012.

28. Doege K, Grajecki D, Zyriax BC, Detinkina E, Zu Eulenburg C and Buhling $\mathrm{KJ}$ : Impact of maternal supplementation with probiotics during pregnancy on atopic eczema in childhood - a meta-analysis. Br J Nutr 107: 1-6, 2012.

29. Garcia-Larsen V, Ierodiakonou D, Jarrold K, Cunha S, Chivinge J, Robinson Z, Geoghegan N, Ruparelia A, Devani P, Trivella M, et al: Diet during pregnancy and infancy and risk of allergic or autoimmune disease: A systematic review and meta-analysis. PLoS Med 15: e1002507, 2018.

30. Foolad N, Brezinski EA, Chase EP and Armstrong AW: Effect of nutrient supplementation on atopic dermatitis in children: A systematic review of probiotics, prebiotics, formula, and fatty acids. JAMA Dermatol 149: 350-355, 2013.

31. Zhao M, Shen C and Ma L: Treatment efficacy of probiotics on atopic dermatitis, zooming in on infants: A systematic review and meta-analysis. Int J Dermatol 57: 635-641, 2018.

32. Björkstén B, Sepp E, Julge K, Voor T and Mikelsaar M: Allergy development and the intestinal microflora during the first year of life. J Allergy Clin Immunol 108: 516-520, 2001. 
33. Murray CS, Tannock GW, Simon MA, Harmsen HJ, Welling GW, Custovic A and Woodcock A: Fecal microbiota in sensitized wheezy and non-sensitized non-wheezy children: A nested case-control study. Clin Exp Allergy 35: 741-745, 2005.

34. Huang R, Ning H, Shen M, Li J, Zhang J and Chen X: Probiotics for the treatment of atopic dermatitis in children: A systematic review and meta-analysis of randomized controlled trials. Front Cell Infect Microbiol 7: 392, 2017.

35. Kim SO, Ah YM, Yu YM, Choi KH, Shin WG and Lee JY: Effects of probiotics for the treatment of atopic dermatitis: A meta-analysis of randomized controlled trials. Ann Allergy Asthma Immunol 113: 217-226, 2014.

36. Navarro-López V, Ramírez-Boscá A, Ramón-Vidal D, Ruzafa-Costas B, Genovés-Martínez S, Chenoll-Cuadros E, Carrión-Gutiérrez M, Horga de la Parte J, Prieto-Merino D and Codoñer-Cortés FM: Effect of oral administration of a mixture of probiotic strains on SCORAD index and use of topical steroids in young patients with moderate atopic dermatitis: A randomized clinical trial. JAMA Dermatol 154: 37-43, 2018

37. Roessler A, Friedrich U, Vogelsang H, Bauer A, Kaatz M, Hipler UC Schmidt I and Jahreis G: The immune system in healthy adults and patients with atopic dermatitis seems to be affected differently by a probiotic intervention. Clin Exp Allergy 38: 93-102, 2008.

38. Yoshida Y, Seki T, Matsunaka H, Watanabe T, Shindo M, Yamada $\mathrm{N}$ and Yamamoto $\mathrm{O}$ : Clinical effects of probiotic Bifidobacterium breve supplementation in adult patients with atopic dermatitis. Yonago Acta Med 53: 37-45, 2010.

39. Drago L, Iemoli E, Rodighiero V, Nicola L, De Vecchi E and Piconi S: Effects of Lactobacillus salivarius LS01 (DSM 22775) treatment on adult atopic dermatitis: A randomized placebo-controlled study. Int J Immunopathol Pharmacol 24 1037-1048, 2011

40. Iemoli E, Trabattoni D, Parisotto S, Borgonovo L, Toscano M, Rizzardini G, Clerici M, Ricci E, Fusi A, De Vecchi E, et al: Probiotics reduce gut microbial translocation and improve adult atopic dermatitis. J Clin Gastroenterol 46 (Suppl 1): S33-S40, 2012.

41. Matsumoto M, Ebata T, Hirooka J, Hosoya R, Inoue N, Itami S, Tsuji K, Yaginuma T, Muramatsu K, Nakamura A, et al: Antipruritic effects of the probiotic strain LKM512 in adults with atopic dermatitis. Ann Allergy Asthma Immunol 113: 209-216.e7, 2014.

42. Maslowski KM, Vieira AT, Ng A, Kranich J, Sierro F, Yu D, Schilter HC, Rolph MS, Mackay F, Artis D, et al: Regulation of inflammatory responses by gut microbiota and chemoattractant receptor GPR43. Nature 461: 1282-1286, 2009.

43. De Preter V, Geboes K, Verbrugghe K, De Vuyst L, Vanhoutte T, Huys G, Swings J, Pot B and Verbeke K: The in vivo use of the stable isotope-labelled biomarkers lactose- $\left[{ }^{15} \mathrm{~N}\right]$ ureide and $\left[{ }^{2} \mathrm{H}_{4}\right]$ tyrosine to assess the effects of pro- and prebiotics on the intestina flora of healthy human volunteers. Br J Nutr 92: 439-446, 2004.

44. Schley PD and Field CJ: The immune-enhancing effects of dietary fibres and prebiotics. Br J Nutr 87 (Suppl 2): S221-S230, 2002.

45. Passeron T, Lacour JP, Fontas E and Ortonne JP: Prebiotics and synbiotics: Two promising approaches for the treatment of atopic dermatitis in children above 2 years. Allergy 61: 431-437, 2006.

46. Enomoto T, Sowa M, Nishimori K, Shimazu S, Yoshida A Yamada K, Furukawa F, Nakagawa T, Yanagisawa N, Iwabuchi N, et al: Effects of bifidobacterial supplementation to pregnant women and infants in the prevention of allergy development in infants and on fecal microbiota. Allergol Int 63: 575-585, 2014.
47. Nwanodi O: Skin protective nutraceuticals: The current evidence in brief. Healthcare (Basel) 6: 40, 2018.

48. Feleszko W, Jaworska J, Rha RD, Steinhausen S, Avagyan A, Jaudszus A, Ahrens B, Groneberg DA, Wahn U and Hamelmann E: Probiotic-induced suppression of allergic sensitization and airway inflammation is associated with an increase of $\mathrm{T}$ regulatorydependent mechanisms in a murine model of asthma. Clin Exp Allergy 37: 498-505, 2007.

49. Jang SO, Kim HJ, Kim YJ, Kang MJ, Kwon JW, Seo JH, Kim HY, Kim BJ, Yu J and Hong SJ: Asthma prevention by Lactobacillus rhamnosus in a mouse model is associated with CD4(+)CD25(+) Foxp3(+) T cells. Allergy Asthma Immunol Res 4: 150-156, 2012.

50. Radulian G: Alimentele si sanatatea. In: Nutritie si Sanatate Dentara. Editura Universitara Carol Davila, Bucuresti, pp276, 2008 (In Romanian).

51. Zheng J, Feng Q, Zheng S and Xiao X: The effects of probiotics supplementation on metabolic health in pregnant women: An evidence based meta-analysis. PLoS One 13: e0197771, 2018

52. Kim HJ, Kim HY, Lee SY, Seo JH, Lee E and Hong SJ: Clinical efficacy and mechanism of probiotics in allergic diseases. Korean J Pediatr 56: 369-376, 2013

53. Messaoudi M, Lalonde R, Violle N, Javelot H, Desor D, Nejdi A, Bisson JF, Rougeot C, Pichelin M, Cazaubiel M, et al: Assessment of psychotropic-like properties of a probiotic formulation (Lactobacillus helveticus R0052 and Bifidobacterium longum R0175) in rats and human subjects. Br J Nutr 105: 755-764, 2011.

54. Akay HK, Bahar Tokman H, Hatipoglu N, Hatipoglu H, Siraneci R, Demirci M, Borsa BA, Yuksel P, Karakullukcu A, Kangaba AA, et al: The relationship between bifidobacteria and allergic asthma and/or allergic dermatitis: A prospective study of 0-3 years-old children in Turkey. Anaerobe 28: 98-103, 2014.

55. Margină D, Olaru OT, Ilie M, Grădinaru D, GuTu C, Voicu S, Dinischiotu A, Spandidos DA and Tsatsakis AM: Assessment of the potential health benefits of certain total extracts from Vitis vinifera, Aesculus hyppocastanum and Curcuma longa. Exp Ther Med 10: 1681-1688, 2015.

56. Margina D, Ilie M, Manda G, Neagoe I, Danciulescu-Miulescu R, Purdel CN and Gradinaru D: In vitro effects of prolonged exposure to quercetin and epigallocatechin gallate of the peripheral blood mononuclear cell membrane. Cell Mol Biol Lett 19: 542-560, 2014.

57. Ruan Y, Sun J, He J, Chen F, Chen R and Chen H: Effect of probiotics on glycemic control: A systematic review and meta-analysis of randomized, controlled trials. PLoS One 10: e0132121, 2015.

58. Mishra V, Shah C, Mokashe N, Chavan R, Yadav H and Prajapati J: Probiotics as potential antioxidants: A systematic review. J Agric Food Chem 63: 3615-3626, 2015.

59. Ma YY, Li L, Yu CH, Shen Z, Chen LH and Li YM: Effects of probiotics on nonalcoholic fatty liver disease: A meta-analysis. World J Gastroenterol 19: 6911-6918, 2013.

60. He J,Zhang F and Han Y: Effect of probiotics on lipid profiles and blood pressure in patients with type 2 diabetes: A meta-analysis of RCTs. Medicine (Baltimore) 96: e9166, 2017.

61. Zhang A and Silverberg JI: Association of atopic dermatitis with being overweight and obese: A systematic review and metaanalysis. J Am Acad Dermatol 72: 606-16e4, 2015.

62. Radtke MA, Schäfer I, Glaeske G, Jacobi A and Augustin M: Prevalence and comorbidities in adults with psoriasis compared to atopic eczema. J Eur Acad Dermatol Venereol 31: 151-157, 2017. 